Rev. Saúde públ., S. Paulo, 19:146-53, 1985

\title{
FOCO DE LEISHMANIOSE TEGUMENTAR AMERICANA NOS MUNICIPIOS DE VIANA E CARIACICA, ESTADO DO ESPIRITO SANTO, BRASIL*
}

\author{
Gelcilio Coutinho Barros** \\ Paulo Augusto Sessa** \\ Enilse Antunes de Mattos** \\ Valquiria Rocha Daher Carias** \\ Wilson Mayrink*** \\ José Tasso Aires de Alencar**** \\ Aloisio Falqueto***** \\ Antonio Claudino de Jesus**
}

BARROS, G.C. et al. Foco de leishmaniose tegumentar americana nos municipios de Viana

e Cariacica, Estado do Espirito Santo, Brasil. Rev. Saúde públ., S. Paulo, $19: 146-53,1985$.

RESUMO: Descreve-se um foco de leishmaniose tegumentar americana abrangendo quatro localidades dos municípios de Viana e Cariacica situados próximo ao litoral do Estado do Espírito Santo, Brasil. Foram diagnosticados 71 casos no período de maio de 1978 a dezembro de 1982. A confirmação laboratorial foi obtida pela intradermorreação de Montenegro em 66 pacientes e pelo encontro do parasita em 15. A faixa etária mais acometida foi a de 0-10 anos. Amostras do parasita isoladas de 3 pacientes e de 27 cães foram classificadas como Leishmania braziliensis braziliensis. De apenas dois Proechimys iheringi, entre 257 mamíferos silvestres estudados, isolou-se Leishmania, que foi identificada como Leishmania mexicana aristedesi. As espécies de flebotomíneos mais numerosas (Lutzomyia intermedia, $L$. fischeri e $L$. migonei) apresentaram um elevado grau de adaptação aos ambientes intra e peri-domibiliar. Levantou-se a hipótese da transmissão estar ocorrendo no intra e peri-domicílio. ziliensis.

UNITERMOS: Leishmaniose tegumentar, ocorrência, Leishmania braziliensis bra-

\section{INTRODUÇÃO}

A existência da Leishmaniose tegumentar americana no Espírito Santo foi primeiramente registrada por Cunha ${ }^{4}(1912)$ e Carini ${ }^{3}$ (1912), e posteriormente por Terra ${ }^{15}$ (1913) e Silva ${ }^{14}$ (1915). Daí em diante não foram encontradas referências bibliográficas sobre esta moléstia no Estado, apesar de ser conhecida dos clíni- cos, principalmente dermatologistas e otorrinolaringologistas, como foi possível constatar. Dados obtidos dos registros na Superintendência de Campanhas do Ministério da Saúde - Regional do Espírito Santo (SUCAM-ES) que indicam sua ocorrência no Estado.

Em fins de 1977 passou-se a observar

- Trabalho parcialmente apresentado no XVI Congresso da Sociedade Brasileira de Medicina Tropical, Natal, RN. 1980; realizado com auxílio do Conselho Nacional de Desenvolvimento Clentifico e Tecnológico (CNPq) - Proc. no 2222-8-119/78 e da Superintendência de Campanhas do Ministério da Saúde - Regional do Espirito Santo (SUCAM-ES).

* Do Departamento de Patologia do Centro Biomédico da Universidade Federal do Espírito Santo - Av. Marechal Campos, 1468, Maruípe - 29000 - Vitória, ES - Brasil.

* Do Departamento de Parasitologia do Instituto de Ciências Biológicas da Universidade Federal de Minas Gerais - Caixa Postal 2486 - 30000 - Belo Horizonte, MG - Brasil.

*** Da Superintendêncla de Campanhas do Ministério da Saúde - Regional do Espírito Santo (SU. CAM-ES) - Rua Moacir Strauch s/no - Praia do Canto - 29000 - Vitória, ES - Brasil

**** Do Departamento de Medicina Social do Centro Biomédico da Universidade Federal do Espirito Santo - 29000 - Vitória, ES - Brasil. 
BARROS, G.C. et al. Foco de leishmaniose tegumentar americana nos municípios de Viana e Cariacica, Estado do Espírito Santo, Brasil. Rev. Saúde públ., S. Paulo, 19:146-53. 1985.

um crescente número de casos com intradermorreação de Montenegro positiva, atendidos no Hospital Universitário Cassiano Antonio Moraes da Universidade Federal do Espírito Santo, sendo a maioria procedente das localidades de Povoação, Perobas e Coaca no município de Viana, e de Formate situada nos municípios de Viana e Cariacica. Segundo informações obtidas da SUCAM-ES, entre janeiro de 1973 a abril de 1978 haviam sido diagnosticados 39 casos naquela região.

Como essas localidades apresentavam profundas alterações ambientais e não se configuravam como de colonização recente, planejou-se o presente trabalho que se constituiu na descrição da área e na investigação clínica dos casos humanos ocorridos no período de maio de 1978 a dezembro de 1982 . O estudo da fauna flebotomínica e dos reservatórios foi realizado em trabalhos paralelos por Mattos ${ }^{8}$ (1981) e Falqueto ${ }^{5}$ (1984).

\section{MATERIAL E METODOS}

Foram escolhidas as localidades de Povoação, Perobas, Coaca e Formate em função do número de casos de leish-

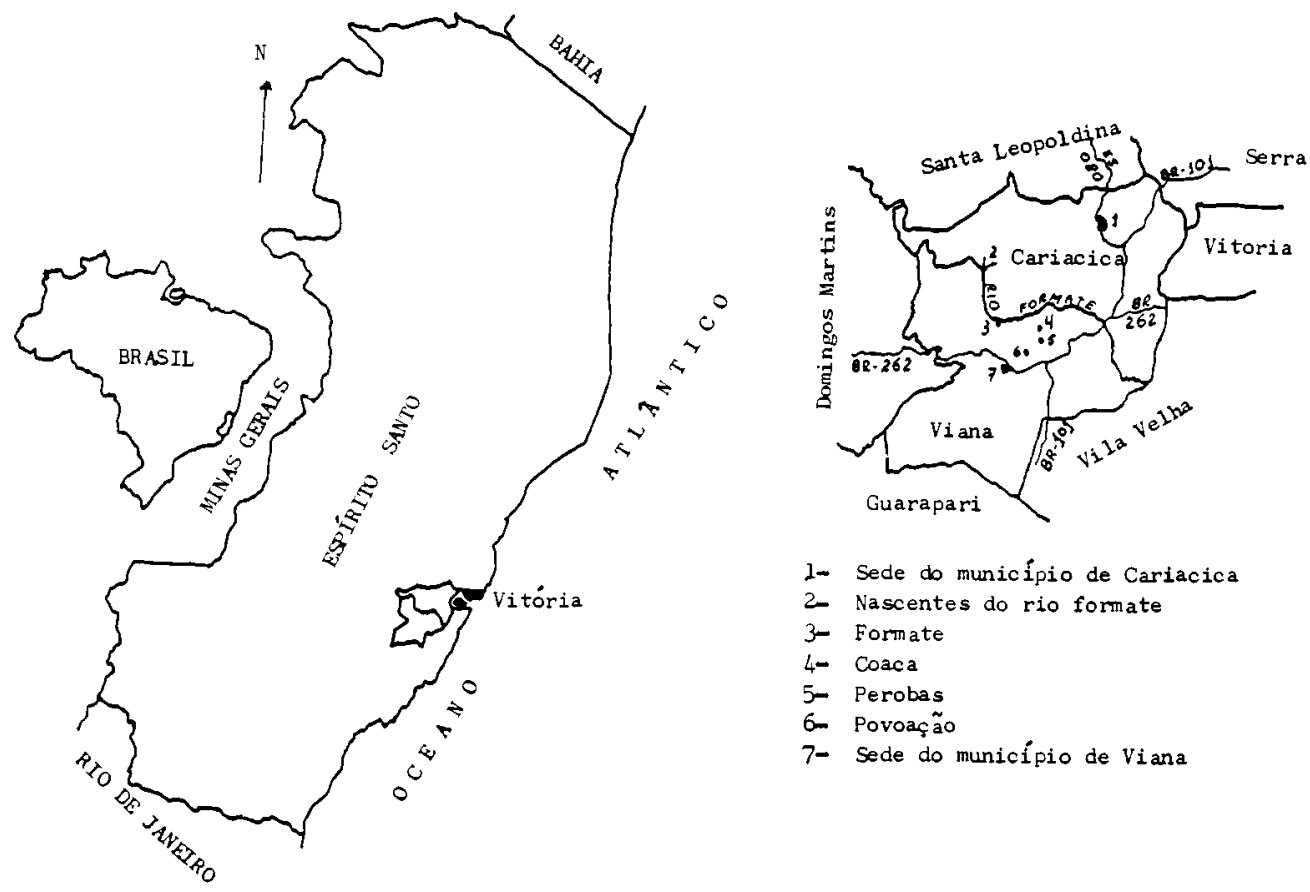

Fig. I - Poslç«o geograflca das localidades de Povoaçāo, Perobas, Coaca e Formate nos mu nicípios de Viana e Cariacica, Estado do Espírito Santo, Brasil.

maniose tegumentar americana que nelas foram inicialmente diagnosticados. As três primeiras situam-se no município de Viana enquanto que Formate é cortada pelo rio do mesmo nome que separa aquele município do de Cariacica. Essas localidades estão a uma latitude de $20^{\circ}$ 23 ' 14 " sul e longitude de $40^{\circ} 25^{\prime} 32^{\prime \prime}$ a 
BARROS, G.C. et al. Foco de leishmaniose tegumentar americana nos municípios de Viana e Cariacica, Estado do Espírito Santo, Brasil. Rev. Saúde públ., S. Paulo, 19:146-53. 1985.

oeste do meridiano de Greenwich. Distam aproximadamente $20 \mathrm{~km}$ da cidade de Vitória, capital do Estado do Espírito Santo (Fig. 1), fazendo parte da Microrregião Estadual 5-Vitória. O clima é tropical com pluviosidade máxima ocorrendo entre os meses de dezembro e fevereiro, e o período seco de julho a agosto. E uma região contínua, muito acidentada em alguns trechos, constituída de serras cujos morros determinam a formação de vales estreitos, quentes e úmidos. Segundo Ballestrero ${ }^{2}$ (1951), o predomínio era, fito-fisionomicamente, o de mata atlântica que foi substituída por cafezais numa boa extensão, e estes por sua vez, desde 1945 , vem gradativamente sendo substituídos por bananais. Da fauna silvestre de vertebrados destacamse roedores e marsupiais. Como animais domésticos predominam o cão, a galinha, o porco e o gato, segundo a ordem citada.

De acordo ainda com Ballestrero ${ }^{2}$ (1951), a população da área é constituída de brancos descendentes de alemães e italianos, de negros e de mestiços. Um levantamento realizado pela SUCAM-ES, em 1978, evidenciou um total de 632 habitantes, sendo que 116 residiam em Povoação, 124 em Perobas, 178 em Coaca e 214 em Formate. Do total, 224 tinham idade de $0-10$ anos, 151 de $11-20$ e 257 acima de 20 anos. A situação sócio-econômica da população é precária, sem atendimentos básicos de infra-estrutura sanitária, e com baixa renda per capita. A economia da região é agropastoril sendo que os homens se ocupam, em sua maioria, da bananicultura. As habitações são, em geral, rústicas e pobres, construídas de barro batido, madeira e alvenaria, em meio aos bananais. A água utilizada provém de pequenos córregos em cujas proximidades localizam-se as habitações.

\footnotetext{
* Localizado no Rio de Janeiro, RJ - Brasil.
}

O diagnóstico de leishmaniose foi estabelecido com base no aspecto clínico das lesões, através da intradermorreação de Montenegro (IRM), e pelo exame de impressões em lâminas e cortes histológicos preparados a partir de fragmentos de pele obtidos por biópsia. Para a execução da IRM utilizou-se antígeno padronizado por Melo e col. ${ }^{9}$ (1977) e o critério de leitura foi o estabelecido por esses mesmos autores.

$\mathrm{O}$ isolamento do parasita para posterior classificação foi feito por inoculação em hamster de triturado de tecido obtido por biópsia.

Para o estudo da fauna flebotomínica realizaram-se capturas semanais sistematizadas, no período de maio de 1979 a abril de 1980, em 4 ambientes distintos: residências, galinheiros, bananais e matas adjacentes, conforme descrição de Mattos $^{8}$ (1981).

O estudo dos reservatórios constou de capturas semanais sistematizadas nos bananais e matas, e exame de todos os mamíferos domésticos (cães, gatos, equinos e muares), executado por Falqueto ${ }^{5}$ (1984) no período de novembro de 1981 a outubro de 1982. A demonstração do parasita foi feita pelo exame de impressões em lâminas preparadas com fragmentos de pele e vísceras, e pela inoculação de triturado de tecido em hamster.

A classificação das amostras de Leishmania isoladas foi realizada pelo método dos anticorpos monoclonais, no Instituto Oswaldo Cruz.*

\section{RESULTADOS}

A distribuição anual dos 71 casos de leishmaniose tegumentar americana, registrados por localidade, faixa etária e sexo, está na Tabela 1.

Em 68 pacientes as lesões eram exclusivamente cutâneas e, em 3 , apenas 
BARROS, G.C. et al. Foco de leishmaniose tegumentar americana nos municípios de Viana e Cariacica, Estado do Espírito Santo, Brasil. Rev. Saúde públ., S. Paulo, 19:146-53. 1985.

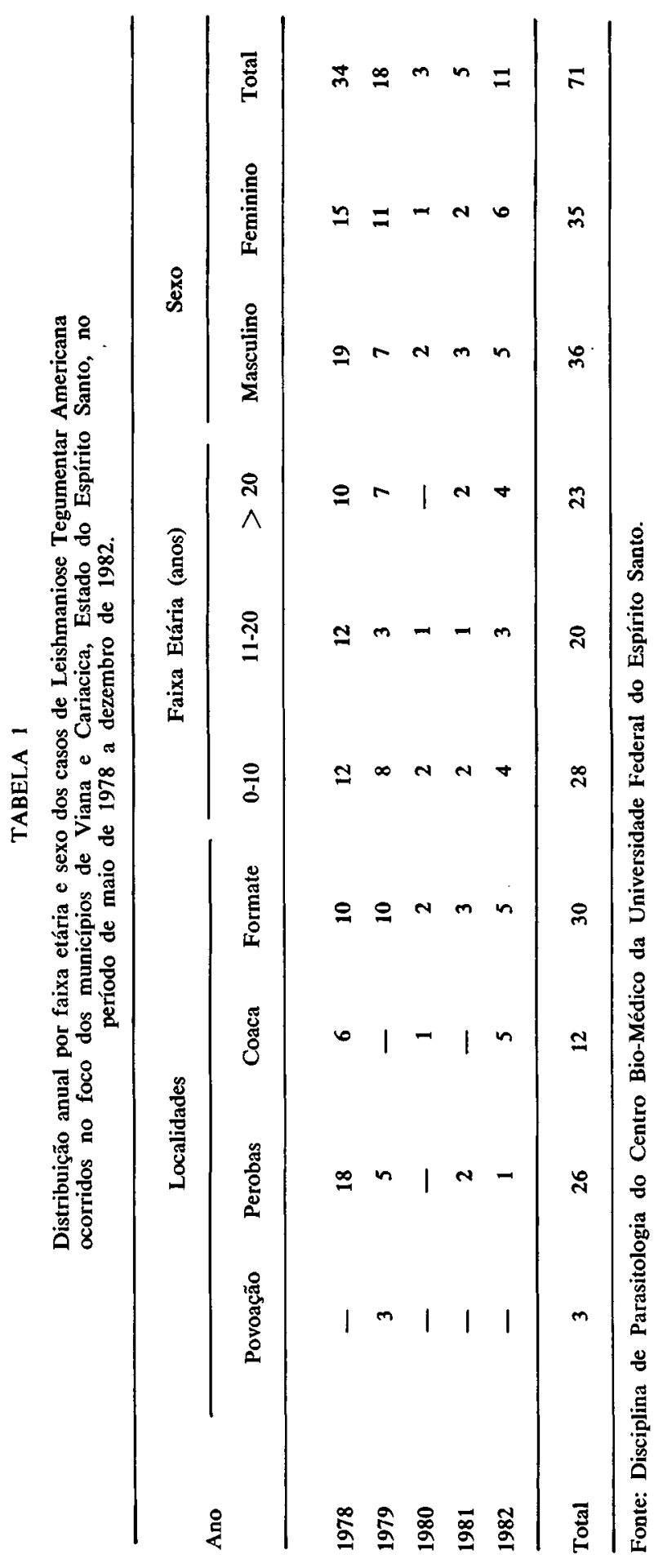


BARROS, G.C. et al. Foco de leishmaniose tegumentar americana nos municípios de Viana e Cariacica, Estado do Espírito Santo, Brasil. Rev. Saúde públ., S. Paulo, 19:146-53. 1985.

de mucosa nasal. Dentre os primeiros o tempo decorrido desde a percepção da lesão pelo paciente até o dia do primeiro atendimento médico foi igual ou menor a 150 dias em 46, maior que este tempo em 7, e informado com imprecisão em 15. A grande maioria (65) apresentava até 3 lesões. Não foi cbservado comprometimento linfático. Nos 3 pacientes com lesão de mucosa nasal a evolução era de 20 dias em um, de 3 anos em outro e não precisado pelo terceiro.

A IRM resultou positiva em 66 $(92,95 \%)$ dos 71 pacientes com áreas nunca inferiores a $0,2 \mathrm{~cm}^{2}(5 \mathrm{~mm}$ de diâmetro). A biópsia executada nos 5 com IRM negativa revelou a presença do parasita em todos. A biópsia mostrou-se também positiva em 10 de 13 outros pacientes portadores da forma cutânea. As amostras do parasita isoladas de 3 desses pacientes foram identificadas como Leishmania braziliensis braziliensis.

Foram capturados 52.248 exemplares de flebotomíneos sendo que Lutzomyia intermedia, $L$. fischeri e $L$. migonei representaram em conjunto $98,37 \%$ do total. A densidade foi maior no domicílio e galinheiro, intermediária no bananal e menor na mata.

Entre os mamíferos domésticos a presença do parasita foi comprovada em 32 cães $(17,2 \%)$ dos 186 existentes na área. As 27 amostras isoladas foram classificadas também como $L$. braziliensis braziliensis.

Foram capturados 257 mamíferos silvestres pertencentes a 3 ordens (Rodentia, Marsupialia e Lagomorfa), englobando 5 famílias com um total de 15 espécies. De apenas dois Proechimys iheringi isolou-se a Leishmania cujas amostras foram identificadas como Leishmania mexicana aristedesi.

A Tabela 2 mostra a distribuição dos casos registrados pela SUCAM-ES, no período de janeiro de 1973 a abril de 1978 , de acordo com o ano e a procedência.

TABELA 2

Distribuição anual dos casos de Leishmaniose tegumentar americana registrados no foco dos municípios de Viana e Cariacica, Estado do Espírito Santo, no período de janeiro de 1973 a abril de 1978 .

\begin{tabular}{ccccc}
\hline & \multicolumn{3}{c}{ Localidades } \\
\cline { 2 - 4 } Ano & Perobas & Coaca & Formate & Total \\
\hline 1973 & 5 & - & 1 & 6 \\
1974 & 6 & - & 2 & 8 \\
1975 & 6 & 1 & 2 & 9 \\
1976 & 1 & - & 1 & 2 \\
1977 & 10 & - & - & 10 \\
1978 & 9 & 1 & - & 10 \\
\hline Total & 37 & 2 & 6 & 45 \\
\hline
\end{tabular}

Fonte: Superintendência de Campanhas do Ministério da Saúde - Regional do Espírito Santo. 
BARROS, G.C. et al. Foco de leishmaniose tegumentar americana nos municípios de Viana

e Cariacica, Estado do Espírito Santo, Brasil. Rev. Saúde públ., S. Paulo, 19:146-53. 1985.

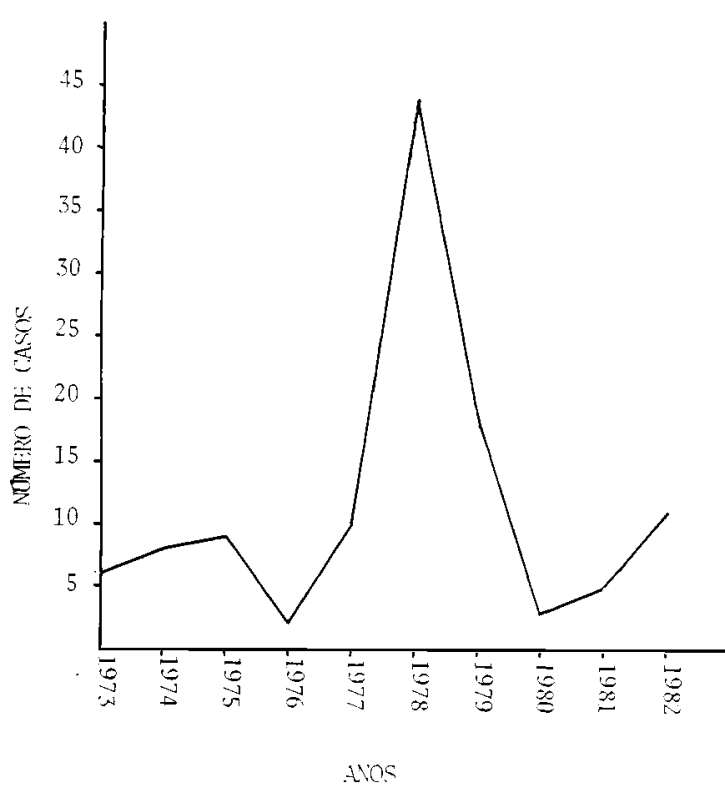

Fig. 2 - Distribuição anual dos casos humanos de leishmaniose tegumentar americana registrados no foco dos municípios de Viana e Cariacica, Estado do Espírito Santo, no período de janeiro de 1973 a abril de 1982 .

Fonte: Superintendência de Campanhas do Ministério da Saúde - Regional do Espírito Santo e Disciplina de Parasitologia do Centro Bio-Médico da Universidade Federal do Espírito Santo.

Na Fig. 2 está o número total de casos ocorridos na área desde janeiro de 1973 a dezembro de 1982.

\section{DISCUSSĀO E CONCLUSÕES}

Contrariando a idéia dominante de que a leishmaniose tegumentar americana ocorre em casos isolados em várias partes do Espírito Santo, verificou-se a existência de um foco autóctone de transmissão ativa. Levando-se em conta que a população da área é relativamente estável, observou-se que em menos de cinco anos a doença, ao atingir 71 pessoas, alcançou $11,23 \%$ dessa população (Tabela 1). Os dados obtidos da SUCAM-
ES, que registrou 45 casos em 3 das 4 localidades no período de 1973 a abril de 1978, mostram que a área vem se comportando como endêmica há alguns anos. A análise conjunta desses dados torna evidente a ocorrência de um surto epidêmico no ano de 1978 , atingindo 44 pessoas.

Constatou-se que nas localidades estudadas a leishmaniose tegumentar americana, não se reveste de características puramente profissionais como observado recentemente por Silva e col. ${ }^{13}$ (1979) na colonização agrícola de Buriticupu, no Maranhão, e classicamente descrita por Pessôa e Martins ${ }^{11}$ (1977). A devastação da mata primitiva e sua substituição pelo bananal, em meio ao qual os habitantes frequientemente constroem suas casas, parece ter criado um ambiente favorável ao desenvolvimento de flebotomíneos. Nos domicílios e instalações peridomiciliares os insetos encontraram abrigo e alimentação farta representada pelos moradores e animais domésticos. O aparecimento de leishmaniose tegumentar americana em áreas de plantio de banana vem se tornando frequiente em áreas litorâneas do Brasil, visto o relato de Forattini $^{6}$ (1973), no Estado de São Paulo, e os de Menezes ${ }^{10}$ (1976), Araújo Filho ${ }^{1}$ (1978) e Sabroza ${ }^{12}$ (1983), no Estado do Rio de Janeiro:

A maior prevalência da doença na faixa de 0-10 anos, o acometimento indiferenciado quanto ao sexo, a elevada taxa de infecção dos cães (a mais alta já registrada na literatura), a captura de flebotomíneos pertencentes a espécies já incriminadas na transmissão e a alta densidade dessas espécies no domicílio e peridomicílio, levam a admitir que a transmissão esteja ocorrendo nesses dois ambientes.

A infecção do homem e dos cães por parasita identificado como L. braziliensis braziliensis e o encontro de apenas dois Proechimys iheringi infectados com 
BARROS, G.C. et al. Foco de leishmaniose tegumentar americana nos municípios de Viana e Cariacica, Estado do Espírito Santo, Brasil. Rev. Saúde públ., S. Paulo, 19:146-53. 1985.

a $L$. mexicana aristedesi, espécie que foi isolada por Herrer e col. ${ }^{7}$ (1971) de animais silvestres no Panamá, levam a pensar na existência de dois ciclos: um envolvendo o domicílio e instalações peridomiciliares, e o outro o ambiente silvestre.

Pelas características observadas supõe-se que a leishmaniose tegumentar americana nas localidades de Povoação, Perobas, Coaca e Formate esteja se comportando como uma zoonose em que o cão seria o reservatório mais importante, a despeito da constante pobreza de parasitas nas lesões. Tal hipótese não afasta a possibilidade de que o homem e o cão, compartilhando os mesmos ambientes, possam estar desempenhando papéis epidemiológicos semelhantes. Novos estudos estão sendo realizados na área com o sentido de trazer esclarecimentos a algumas das hipóteses formuladas.

BARROS, G.C. et. al. [A focus of mucocutaneous leishmaniasis in Viana and Cariacica counties, Espírito Santo State, Brazil]. Rev. Saúde públ., S. Paulo, $19: 146-53,1985$.

ABSTRACT: A mucocutaneous leishmaniasis focus occuring in four village situated in Viana and Cariacica counties, located near the sea-coast of Espírito Santo State, Brazil, is described. Seventy one cases of the disease were diagnosed between May 1978 and December 1982. The laboratorial confirmation was obtained by the Montenegro test in 66 patients and by biopsy in 15. The 0 to 10 year age-group was the most affected. The strains of the parasites isolated from 3 patients and 27 dogs were classified as Leishmania braziliensis braziliensis. Among 257 wild animals captured the Leishmania was isolated in two Proechimys iheringi only, and identified as $L$. mexicana aristedesi. The most numerous sandfly specimens (Lutzomyia intermedia, $L$. fischeri and $L$. migonei) were captured with greater frequency both inside and outside residences. The possibility of the transmission's occuring both inside and outside residences was noteworthy.

UNITERMS: Leishmaniasis, mucocutaneous occurence. Leishmania braziliensis braziliensis.

\section{REFERENCIAS BIBLIOGRÄFICAS}

1. ARAUJO FILHO, N.A. Epidemiologia da leishmaniose tegumentar americana na Ilha Grande, Rio de Janeiro. Estudos sobre a infecção humana, reservatórios e transmissores. Rio de Janeiro, 1978. [Dissertação de Mestrado - Universidade Federal do Rio de Janeiro]

2. BALLESTRERO, H.L. Subsídios para o estudo da geografia e da história do município de Viana, Jabaeté, Espírito Santo. Vitória, Escola Técnica de Vitória, 1951.

3. CARINI, A. Um novo caso de leishmaniose das mucosas. Arch. Soc. Med. Cir. S. Paulo, 14:364-6, 1912.

4. CUNHA, C. Comunicação à sessão de 28 de julho da Sociedade Brasileira de Dermatologia. Bol. Soc. bras. Der matol., 1:42, 1912.
5. FALQUETO, A. Leishmaniose tegumentar em Viana, Estado do Espírito Santo. Investigação sobre infecção natural em animais e sua relação com a ocorrência da doença humana. Rio de Janeiro, 1984. [Dissertação de Mestrado - Universidade Federal do Rio de Janeiro]

6. FORATTINI, O.P. Nota sobre leishmaniose tegumentar no litoral sul do Estado de São Paulo. Rev. Saúde públ., S. Paulo, 7:447-52, 1973

7. HERRER, A.; TELFORD, S.R. \& CHRISTENSEN, H.A. Enzootic cutaneous leishmaniasis in eastern Panama. I . Investigation of the infection among forest mammals, Ann. trop. Med. Parasit., 65:349-58, 1971.

8. MATTOS, E.A. Bionomia dos flebotomíneos de Perobas, município de Viana (ES), área endêmica de leishmanio- 
BARROS, G.C. et al. Foco de leishmaniose tegumentar americana nos municípios de Viana e Cariacica, Estado do Espírito Santo, Brasil. Rev. Saúde públ., S. Paulo, 19:146-53. 1985.

se tegumentar americana. Belo Horizonte, 1981. [Dissertação de Mestrado - Universidade Federal de Minas Gerais]

9. MELO, M.N.; MAYRINK, W.; COSTA, C.A. da; MAGALHÃES, P.A.; DIAS, M.; WILLIAMS, P.; ARAUJO, F.G.; COELHO, M.V. \& BATISTA, S.M. Padronização do antígeno de Montenegro. Rev. Inst. Med. trop. S. Paulo, 19:161-4, 1977 .

10. MENEZES, J.A. Leishmaniose tegumentar no Estado do Rio de Janeiro. Inquéritos por intradermorreação. Rio de Janeiro, 1976. [Dissertação de Mestrado - Faculdade de Medicina UFRJ]

11. PESSOA, S.B. \& MARTINS, A.V. Parasitologia médica. $10 .^{\mathrm{a}} \mathrm{ed}$. Rio de Janeiro, Guanabara Koogan, 1977.

12. SABROZA, P.A. O domicílio como fator de risco na leishmaniose tegumentar americana. Estudo epidemiológico em
Jacarepaguá, município do Rio de Janeiro. Rio de Janeiro, 1983. [Dissertação de Mestrado - Escola Nacional de Saúde Pública]

13. SILVA, A.R.; MARTINS, G.; MELO, J.E.; ARAUJO, J.P.; MENDES, J.R. \& MENDES, M.G. Surto epidèmico de leishmaniose tegumentar americana na colonização agrícola de Buriticupu (Estado do Maranhão), Brasil. Rev. Inst. Med. trop. S. Paulo, 21: 43-50, 1979.

14. SILVA, O.D. Sobre a leishmaniose tegumentar e seu tratamento. Mem. Inst. Oswaldo Cruz, 7:213-48, 1915.

15. TERRA, F. Leishmaniose tégumentaire au Brésil. Bol. Soc. bras. Dermatol., 2(2/3): 58-67, 1913 .

Recebido para publicação em 10/04/1984

Reapresentado em 21/01/1985

Aprovado para publicação em 21/02/1985 